\begin{tabular}{|l|l|l|}
\hline D.Y.-Y. & Yi-Yung & Hsia \\
\hline K. & & Driscoll \\
\hline W. & & Troll \\
\hline W.E. & & Knox \\
\hline
\end{tabular}

From the Cancer Research Institute of the New England Deaconess Hosp.; the Departments of Pediatrics and Biological Chemistry, Harvard Medical School; and the Children's Medical Center, Boston, Mass., U.S.A.

The molecule whose dysfunction is responsible for the hereditary disease of phenylketonuria is the liver enzyme concerned with the oxidation of phenylalanine. Inactivity of this enzyme results in the high blood levels of phenylalanine, the excretion of phenylpyruvic acid, and mental deficiency. Genetic studies have established this condition to be completely recessive, and no related mental or biochemical abnormalities have been found in the heterozygous parents or siblings of the patients. Pauling's introduction of the term "molecular disease" suggests, however, that the fraction of enzyme stemming from the abnormal gene in the heterozygotes should be defective. This prediction is supported by the present findings from phenylalaninetolerance tests that known heterozygotes of phenylketonuria have lower capacities for metabolizing phenylalanine than do normal people.

Plasma levels of L-phenylalanine were determined one, two, and four hours after oral doses of 0.1 g. of L-phenylalanine per kg. body weight, given after an overnight fast, in 19 heterozygous individuals (parents of proved phenylketonuric patients) and an equal number of normal adult controls. L-phenylalanine was measured by a modification of the bacterial decarboxylase method of Udenfriend and Cooper. The fasting plasma phenylalanine levels taken before the test in all individuals were within the range of 0.03 to $0.16 \mu \mathrm{mol} / \mathrm{ml}$. The phenylalanine levels in the heterozygotes during the test were on the average about twice that of 190

Yi-Yung Hsia, Driscoll, Troll and Knox

(648)

the controls at each of the hourly intervals. The distinction between the groups was particularly clear at the fourth hour, and by comparison of the area under the tolerance curve. The test was repeated on several individuals from each group with highly reproducible results.

Table 1. Plasma L-Phenylalanine Levels after L-Phenylalanine Ingestion. Values given are the means + the standard deviation, and the ranges, in $\mu$ mols per ml. of plasma, found in 19 adult controls and 19 parents (heterozygotes) of known phenylketonuric patients.

Hours after Dose 


\section{Sum of Hourly Levels}

Controls: Heterozygotes:

$0.55 \pm .186(0.30-0.90)$

$1.14 \pm .187(0.84-1.44)$

$0.55 \pm .168(0.29-1.02)$

$1.03 \pm .187$

(0.72-1.44)

$0.30 \pm \cdot 076(0.21-0.50)$

$0.76 \pm \cdot 292(0.45-1.62)$

$1.41 \pm .366(0.87-2.19)$

$2.93 \pm .458(2.10-4.02)$

Comparison of the two groups on the basis of age, sex, weight, intelligence, or dose per square meter of body surface failed to reveal discrepancies which could account for the observed differences between the phenylalanine plasma levels of the groups. The possibility was exceedingly small that such a difference could have occurred by chance ( $p$ less than 0.0001 ). The excretion of phenylalanine during the nine hours following the oral dose was determined in about half of the individuals. The amounts excreted were not different in the two groups and were insignificant in relation to the amounts disappearing from the blood during the test period. Phenylpyruvic acid in the urine during the same period was never found in the controls and only in one heterozygote who also showed the highest observed plasma levels of phenylalanine. The most probable interpretation of these results is that the heterozygotes as a group have significantly less than the normal phenylalanine oxidizing enzyme activity. 